\title{
Human pathogenic Mycobacterium kansasii (former subtype I) with zoonotic potential isolated from a diseased indoor pet cat, Japan
}

\section{Hanako Fukano}

National Institute of Infectious Diseases

Tsukasa Terazono

Terazono Animal Hospital Hiroshima, JP

Aki Hirabayashi

National Institute of Infectious Diseases

Mitsunori Yoshida

National Institute of Infectious Diseases

\section{Masato Suzuki}

National Institute of Infectious Diseases

\section{Shinpei Wada}

Nippon Veterinary and Life Science University, Faculty of Veterinary Science Musashino, Tokyo, JP Norihisa Ishii

National Institute of Infectious Diseases

Yoshihiko Hoshino ( $\nabla$ yhoshino@nih.go.jp )

National Institute of Infectious Diseases https://orcid.org/0000-0002-3132-0715

\section{Case Report}

Keywords: Mycobacterium kansasii, Emerging Infectious Diseases, Zoonoses, Diseases Reservoirs, Nontuberculous Mycobacteria

Posted Date: September 28th, 2020

DOl: https://doi.org/10.21203/rs.3.rs-82291/v1

License: (c) (i) This work is licensed under a Creative Commons Attribution 4.0 International License. Read Full License

Version of Record: A version of this preprint was published at Emerging Microbes \& Infections on January 1st, 2021. See the published version at https://doi.org/10.1080/22221751.2021.1878935. 


\section{Abstract}

Mycobacterium kansasii is one of the most prevalent and pathogenic nontuberculous mycobacteria in the world. Herein, we report the first case of $M$. kansasii infection in an indoor domestic cat in Japan. Complete genome sequence analysis of the isolate showed this pathogen is genetically identical to human pathogenic $M$. kansasii.

\section{Introduction}

Nontuberculous mycobacterial (NTM) infections in humans have increased in prevalence in recent decades(1). Most NTM species that are pathogenic to humans are also pathogenic to cats, including Mycobacterium avium, Mycobacterium abscessus, and Mycobacterium xenopi(2). However, little evidence exists of the zoonotic potential of NTM infections.

Mycobacterium kansasii is one of the most prevalent human pathogenic NTM species worldwide and is phylogenetically closest to Mycobacterium tuberculosis(3). Initially, PCR-restriction pattern analysis identified seven subtypes of $M$. kansasii (subtypes I-VII)(4). However, comparative genomic analysis reclassified these subtypes as $M$. kansasii (former subtype I), Mycobacterium persicum (former subtype II), Mycobacterium pseudokansasii (former subtype III), Mycobacterium innocens (former subtype V), and Mycobacterium attenuatum (former subtype VI)(5). Currently, the $M$. kansasii complex (MKC) comprises the M. kansasii, M. persicum, M. pseudokansasii, M. innocens, M. attenuatum and M. gastri species $(5,6)$. These species names will be used throughout this manuscript.

A recent study showed that, compared to other MKC species, $M$. kansasii has ESX-1 type VII secretion system and espACD operon associated with its pathogenicity $(6,7)$. Of the MKC species, $M$. kansasii is the most frequently isolated from patients with pulmonary diseases, while $M$. persicum is associated with immunodeficient HIV-infected patients $(8,9)$. The remaining MKC species are considered non-pathogenic colonizing agents and are typically isolated from tap water samples or animals $(8,9)$. To date, the risk of $M$. kansasii infection and its major environmental reservoir remain poorly understood.

In this study, we report the first case of $M$. kansasii infection in an indoor domestic immunocompetent cat in Japan. Complete genome sequence analysis identified the isolate as human pathogenic $M$. kansasii.

\section{The Study}

The case is a 13-year-old, neutered, female domestic cat weighing $4.0 \mathrm{~kg}$. The cat lived outdoors for the first 2-3 years but has remained indoors only for nearly 10 years. Tests for Feline Leukemia Virus (FeLV) and Feline Immunodeficiency Virus (FIV) were both negative.

Initially, the cat exhibited lid swelling and eye mucus of the left eye. The cat was treated with broadspectrum antibiotics and steroids, but eyelid swelling worsened after 1 to 2 weeks. The swelling site was surgically excised, and the pus drained (Figure 1A). 
Giemsa staining of stamp specimens demonstrated a large number of intracellular non-staining long rod bacilli in macrophages and giant cells (Figure 1B). Histopathological examination revealed pyogranulomatous lesions with neutrophil aggregation and multinucleated giant cell infiltration (Figure 1C). We observed a large amount of acid-fast long rod bacilli by Ziehl-Neelsen staining (Figure 1D).

We used a mycobacterial isolation technique to isolate the strain, referred to as Kuro-I, from the nodular tissue lesion. For long-read and short-read sequencing, we extracted genomic DNA using phenol-

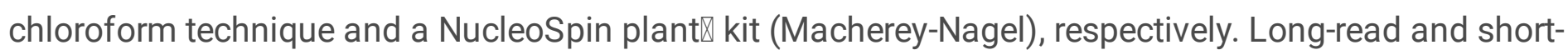
read sequencing were performed on the MinION platform (Oxford Nanopore Technologies, Oxford, UK) and NovaSeq 6000 Platform (Illumina., San Diego, CA). Preparation for the sequencing library and genome assembly using long/short sequenced data is described in the Appendix. The circular genome size of Kuro-l is $6,649,596 \mathrm{bp}$, with a $\mathrm{G}+\mathrm{C}$ content of $66.1 \%$, containing 8,110 putative coding sequences, 3 rRNAs, 54 tRNAs, and 5 CRISPR region.

To compare the Kuro-I strain genome to other MKC species, we obtained next-generation sequencing data for the MKC species from the National Center for Biotechnology Information (NCBI) Assembly and Sequence Read Archive (SRA) databases, and obtained source information from the NCBI BioSample database (Appendix Table). We annotated the genomes with Prokka (Galaxy ver. 1.14.5) and used Roary (Galaxy ver. 3.13.0) for pan-genomic analysis and core-gene alignment, which we used to build a maximum-likelihood phylogenetic tree using RAxML (Galaxy ver.1.0.0), Interactive Tree of Life (iTOL) and Phandango. We calculated the genome-based average nucleotide identity (ANI) with FastANI (Galaxy ver.1.3). We performed pan-genomic analysis for 17 M. kansasii strains by Roary (Galaxy ver. 3.13.0).

Phylogenetic analysis based on core-gene alignments discriminated the MKC species. The Kuro-I strain isolated from the domestic cat and two strains isolated from Rhesus macaques were positioned in the internal clade of $M$. kansasii consist of isolates from pulmonary diseased human (Figure 2). The $17 \mathrm{M}$. kansasii pan-genome has a total 15037 gene; 3100 are shared between the core (3100) and soft-core (0) genes; while 3,767 and 8,170 genes from shell and cloud genes, respectively (Appendix Figure. 1). Coding sequence of espACD operon was present in Kuro-I strain (Appendix Figure. 2).

The ANI values between the Kuro-I strain and the MKC strains M. kansasii $\mathrm{ATCC}^{\mathrm{T}} 12478^{\top}$, M. attenuateum MK41 ${ }^{\top}$, M. pseudokansasii MK142 ${ }^{\top}$, M. gastri DSM43505 ${ }^{\top}$, M. innocens $\mathrm{MK}^{\top} 1^{\top}$, and M. persicum AFPC$000227^{\top}$ were $99.37 \%, 90.38 \%, 92.71 \%, 91.59 \%, 93.43 \%$, and 93.27\%, respectively (Appendix Figure. 3).

The cat received treatment for 6 months with Clarithromycin $(20 \mathrm{mg} / \mathrm{kg}$; BID) and Rifampicin (7 mg/kg; BID), which improved clinical signs, including eyelid swelling and eye mucus in both eyes. There was no recurrence six months after chemotherapy, and the owner was not infected.

\section{Conclusions}

Next-generation sequencing has reclassified the MKC species and revealed pathogenic characteristics(7). Although studies show that the major reservoir of $M$. kansasii infection is tap water instead of 
environmental water sources or soil(10), the infection sources for all of the MKC species remain poorly understood.

M. kansasii has been isolated from a wide variety of animals such as dogs (Canis familiaris), Rhesus Macaque (Macaca mulatta) and cats (Felis catus). However, the respective M. kansasii subtypes and zoonotic potential are unknown(11-13). In this study, we isolated the Kuro-I strain from a diseased indoor domestic cat. Using complete genome sequence analysis, we identified the isolate as $M$. kansasii (former subtype区). The Kuro-I strain was genetically similar to $M$. kansasii isolated from patients with pulmonary diseases and Rhesus macaques. We report the first comparative genomic analysis of $M$. kansasii isolated from animals and humans based on core-gene phylogeny and genome-to-genome distance.

A recent study identified an incidence of cat-to-human transmission of $M$. bovis infection in England, where two people who had close contact with an infected pet cat developed active M. bovis diseases(14). Whole-genome sequencing analysis confirmed cat-to-human transmission for the first time and demonstrated a risk of companion animal associated mycobacterial infection in humans. In this present case, we isolated $M$. kansasii from an immunocompetent domestic cat who has remained indoors for the past decade. This demonstrates that domestic cats, which are among the most common pets, are a susceptible host for $M$. kansasii infection under immunocompetent conditions and could be a reservoir for this emerging pathogen.

In this study, histopathological analysis revealed pyogranulomatous lesions filled with pus containing large amounts of mycobacterial cells. This suggests aerosol transmission capability, which is the primary transmission route for human pulmonary NTM infections, including M. kansasii(14).

Although $M$. kansasii is a drug-sensitive NTM species, inappropriate use of clarithromycin can lead to a high prevalence of macrolide-resistant strains (15). Our data suggest that the treatment of companion animals requires proper antibiotic management by veterinarians to avoid resistance.

In conclusion, we report the first case of $M$. kansasii infection in an indoor pet cat in Japan. This finding suggests that $M$. kansasii has a potential risk of zoonoses and requires the "One Health" approach to control NTM infection. Further studies exploring the environmental sources of $M$. kansasii are necessary to understand the transmission modes or infectious risk of this emerging worldwide disease.

\section{Declarations}

\section{Biographical Sketch}

Dr. Fukano is a senior researcher in the Department of Mycobacteriology, Leprosy Research Centre, National Institute of Infectious Diseases, Tokyo, Japan. Her research interests are antimicrobial resistance and transmission of mycobacteria. 


\section{Acknowledgments}

This work was in part supported by a grant from the Japan Agency for Medical Research and Development/Japan International Cooperation Agency (AMED) to Y. Hoshino囚jp20fk0108064, jp20fk0108075, jp20fk0108093, jp20fk0108129, jp20jm0510004, jp20wm0125007, jp20wm0325003 and jp20wm0225004) and by Grant-in-Aids for Scientific Research (B) and (C) to Y. Hoshino (jp20H02282 and jp18K08312), for Early-Career Scientists to H. Fukano (jp18K15966) and M. Yoshida (jp20K17205) and for Fostering Joint International Research (B) to Y. Hoshino and M. Yoshida (jp19KK0217) from the Japan Society for the Promotion of Science (JSPS) .

\section{References}

1. Lin C, Russell C, Soll B, Chow D, Bamrah S, Brostrom R, et al. Increasing prevalence of nontuberculous mycobacteria in respiratory specimens from US-affiliated pacific Island Jurisdictions. Emerg Infect Dis. 2018;24(3):485-91.

2. Gunn-Moore DA. Feline mycobacterial infections. Vet J. 2014;201(2):230-8.

3. Hoefsloot W, van Ingen J, Andrejak C, Ängeby K, Bauriaud R, Bemer P, et al. The geographic diversity of nontuberculous mycobacteria isolated from pulmonary samples: an NTM-NET collaborative study. Eur Respir J. 2013;42(6):1604-13.

4. Telenti A, Marchesi F, Balz M, Bally F, Böttger EC, Bodmer T. Rapid identification of mycobacteria to the species level by polymerase chain reaction and restriction enzyme analysis. J Clin Microbiol. 1993;31(2).

5. Tagini F, Aeby S, Bertelli C, Droz S, Casanova C, Prod'Hom G, et al. Phylogenomics reveal that Mycobacterium kansasii subtypes are species-level lineages. Description of Mycobacterium pseudokansasii sp. nov., Mycobacterium innocens sp. nov. and Mycobacterium attenuatum sp. nov. Int J Syst Evol Microbiol. 2019;69(6):1696-704.

6. Jagielski T, Borówka P, Bakuła Z, Lach J, Marciniak B, Brzostek A, et al. Genomic insights into the Mycobacterium kansasii complex: An Update. Front Microbiol. 2020;10.

7. Guan Q, Ummels R, Ben-Rached F, Alzahid Y, Amini MS, Adroub SA, et al. Comparative genomic and transcriptomic analyses of Mycobacterium kansasii subtypes provide new insights into their pathogenicity and taxonomy. Front Cell Infect Microbiol. 2020;10.

8. Alcaide F, Benitez MA, Martin R, Bloch KC, Vugia DJ, Reingold AL. Epidemiology of Mycobacterium kansasii [3]. Vol. 131, Annals of Internal Medicine. American College of Physicians; 1999. P. 310-1.

9. Taillard C, Greub G, Weber R, Pfyffer GE, Bodmer T, Zimmerli S, et al. Clinical implications of Mycobacterium kansasii species heterogeneity: Swiss national survey. J Clin Microbiol. 2003;41(3):1240-4.

10. Johnston JC, Chiang L, Elwood K. Mycobacterium kansasii. Microbiol Spectr. 2017;5(1). 
11. Murai A, Maruyama S, Nagata M, Yuki M. Mastitis caused by Mycobacterium kansasii infection in a dog. Vet Clin Pathol. 2013;42(3):377-81.

12. Lee SH, Go DM, Woo SH, Park HT, Kim E, Yoo HS, et al. Systemic Mycobacterium kansasii infection in a domestic shorthair cat. J Comp Pathol. 2017;157(2-3):215-9.

13. Shipley ST, Johnson DK, Roodgar M, Smith DG, Montgomery CA, Lloyd SM, et al. Mycobacterium kansasii isolated from tuberculin-positive Rhesus Macaques (Macaca mulatta) in the absence of disease. Comp Med. 2017;67(4):368-75.

14. O'Connor CM, Abid M, Walsh AL, Behbod B, Roberts T, Booth L V., et al. Cat-to-human transmission of Mycobacterium bovis, United Kingdom. Emerg Infect Dis. 2019;25(12):2284-6.

15. Li Y, Pang Y, Tong X, Zheng H, Zhao Y, Wang C. Mycobacterium kansasii subtype I is associated with clarithromycin resistance in china. Front Microbiol. 2016;7:2097.

\section{Figures}

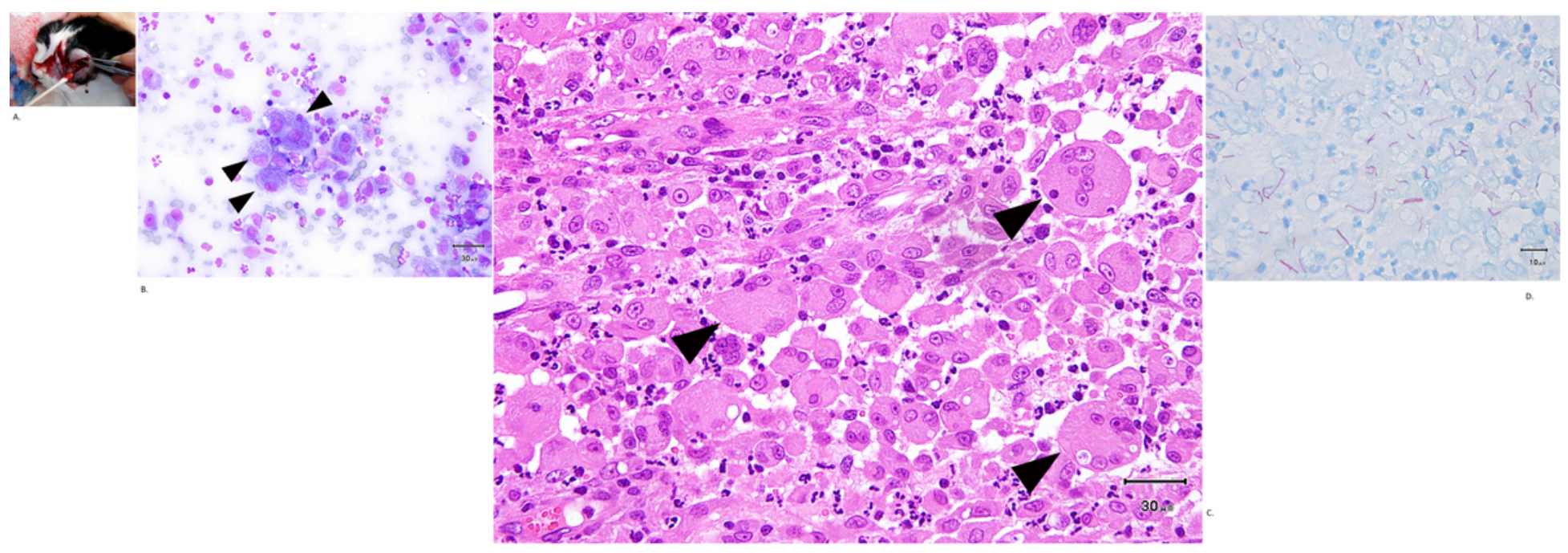

\section{Figure 1}

A) A heavily swollen left eyelid caused by M. kansasii infection in an indoor pet cat. B-D) Results of histopathological analyses. B) Giemsa staining of stamp specimens revealed many intracellular nonstaining long rod bacilli within macrophages or giant cells (arrowheads). C) Hematoxylin and eosin staining demonstrated neutrophil aggregation and multinucleated giant cell or Langhans giant cell infiltration (arrowheads). D) Ziehl-Neelsen staining showed acid-fast long rod bacilli (arrowheads). 


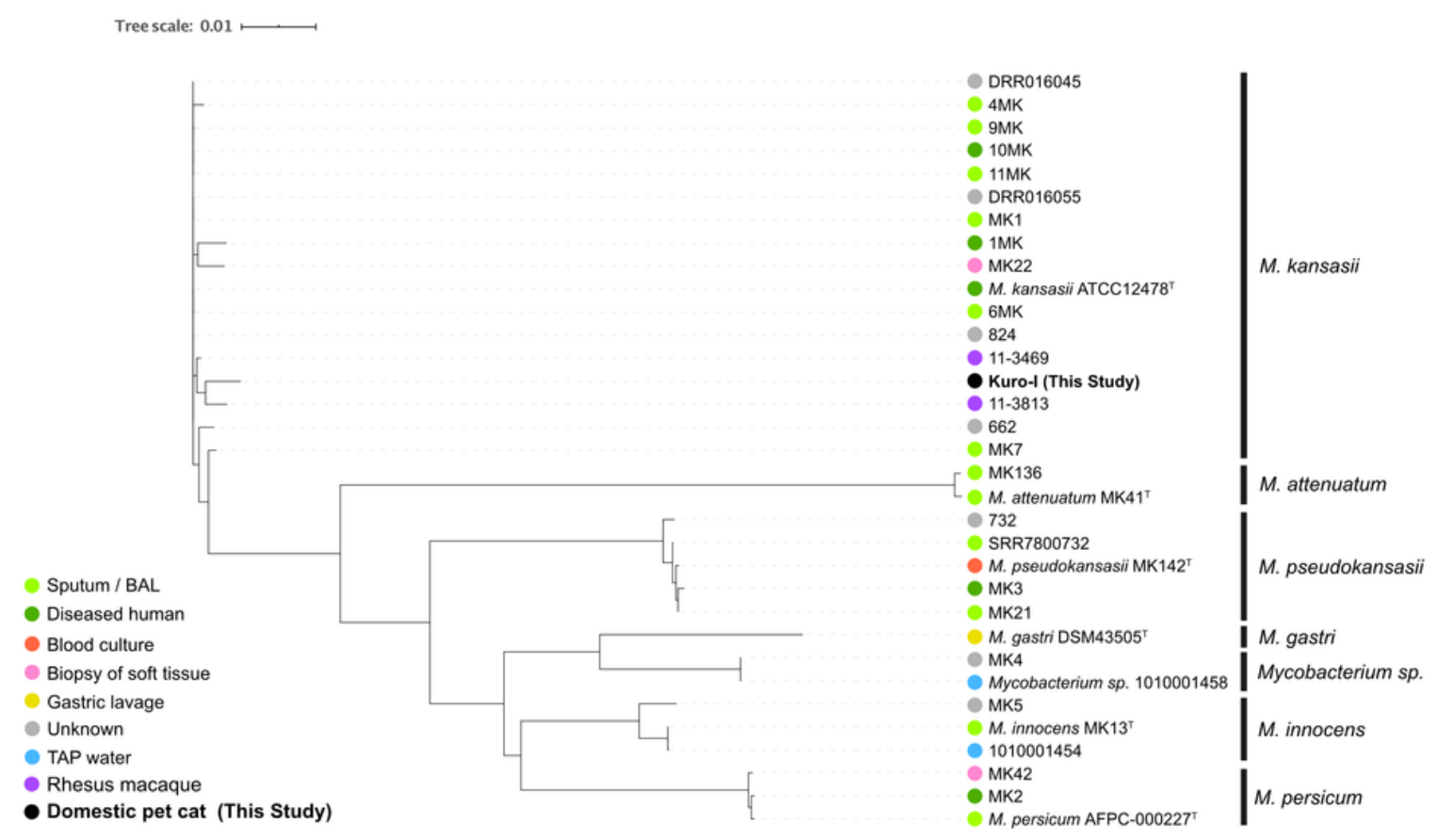

Figure 2

A phylogenetic tree based on core gene alignments of $\mathrm{M}$. kansasii complex (MKC) species. Colored circles indicate the isolate determined by deposited information from the archived NCBI BioSample database.

\section{Supplementary Files}

This is a list of supplementary files associated with this preprint. Click to download.

- TechnicalAppendix.docx

- AppendixFig.1Pangenome.png

- AppendixFig.2ACDoperon.png

- AppendixFig.3ANI.png

- AppendixTable.xlsx 